\title{
UNIFORMITY IN THE POLYNOMIAL WIENER-WINTNER THEOREM
}

\author{
NIKOS FRANTZIKINAKIS
}

\begin{abstract}
In 1993, E. Lesigne proved a polynomial extension of the Wiener-Wintner ergodic theorem and asked two questions: does this result have a uniform counterpart and can an assumption of total ergodicity be replaced by ergodicity? The purpose of this article is to answer these questions, the first one positively and the second one negatively.
\end{abstract}

\section{INTRODUCTION}

The Wiener-Wintner ergodic theorem [5] asserts that if $(X, \mathcal{B}, \mu, T)$ is an ergodic measure preserving system and $f \in L^{1}(\mu)$, then for a.e. $x \in X$ the limit

$$
\lim _{N \rightarrow \infty} \frac{1}{N} \sum_{n=1}^{N} e(n \alpha) \cdot f\left(T^{n} x\right)
$$

exists for every $\alpha \in \mathbb{R}$, where $e(t)=e^{2 \pi i t}$. What makes this a nontrivial strengthening of the Birkhoff ergodic theorem is that the set of full measure for which we have convergence does not depend on the choice of $\alpha \in \mathbb{R}$.

Two different proofs of this theorem are based on the following results which are of interest on their own:

(i) For ergodic systems, if $f \in L^{1}(\mu)$ and $f \in \mathcal{E}_{1}(T)^{\perp}$, then for a.e. $x \in X$ we have that for every $\alpha \in \mathbb{R}$

$$
\lim _{N \rightarrow \infty} \frac{1}{N} \sum_{n=1}^{N} e(n \alpha) \cdot f\left(T^{n} x\right)=0,
$$

where $\mathcal{E}_{1}(T)$ is the set of eigenfunctions of $T$.

(ii) For ergodic systems, if $f \in L^{1}(\mu)$ and $f \in \mathcal{E}_{1}(T)^{\perp}$, then for a.e. $x \in X$ we have

$$
\lim _{N \rightarrow \infty} \sup _{\alpha \in \mathbb{R}}\left|\frac{1}{N} \sum_{n=1}^{N} e(n \alpha) \cdot f\left(T^{n} x\right)\right|=0 .
$$

Condition (ii) is of course stronger than $(i)$.

A natural problem is to find conditions that will allow us to replace the linear polynomials $P(n)=n \alpha$, in (1), (2) , and (3), with higher degree polynomials. This study was 
initiated by Lesigne in [3] and [4. To state his main result we need the notion of level $k$ quasi-eigenfunction, introduced by Abramov ${ }^{1}$ :

Definition 1.1. If $(X, \mathcal{X}, \mu, T)$ is an ergodic system we let $\mathcal{E}_{0}(T)$ denote the set of eigenvalues of $T$ and for $k \in \mathbb{N}$ we define inductively

$$
\mathcal{E}_{k}(T)=\left\{f \in L^{\infty}(\mu):|f|=1 \text { and } T f \cdot \bar{f} \in \mathcal{E}_{k-1}(T)\right\} .
$$

The result of Lesigne is:

Theorem 1.2 (Lesigne [3], 4]). Let $(X, \mathcal{X}, \mu, T)$ be an ergodic system and $f \in L^{1}(\mu)$.

(i) For a.e. $x \in X$, we have for every continuous $\phi: \mathbb{T} \rightarrow \mathbb{C}$, and polynomial $P$ with real coefficient that the averages

$$
\frac{1}{N} \sum_{n=1}^{N} \phi(P(n)) \cdot f\left(T^{n} x\right)
$$

converge as $N \rightarrow \infty$.

(ii) Let $k \in \mathbb{N}$. If $f \in \mathcal{E}_{k}(T)^{\perp}$ and the system is totally ergodic (meaning $T^{r}$ is ergodic for $r \in \mathbb{N}$ ), then for a.e. $x \in X$, we have for every continuous $\phi: \mathbb{T} \rightarrow \mathbb{C}$, and $P \in \mathbb{R}_{k}[t]$ that

$$
\lim _{N \rightarrow \infty} \frac{1}{N} \sum_{n=1}^{N} \phi(P(n)) \cdot f\left(T^{n} x\right)=0
$$

where $\mathbb{R}_{k}[t]$ denotes the set of polynomials with real coefficients and degree at most $k$.

Motivated by the linear case where $f \in \mathcal{E}_{1}(T)^{\perp}$ implies uniform convergence to zero (see equation (3) ), Lesigne asked in [4] whether (ii) has a uniform counterpart. Furthermore, he asked whether the total ergodicity assumption can be removed from (ii). He remarked that this would be the case if one could establish that $\mathcal{E}_{k}(T)^{\perp}=\mathcal{E}_{k}\left(T^{m}\right)^{\perp}$ for every $k, m \in \mathbb{N}$, a result that is easily shown to be true when $k=1$. The purpose of this paper is to answer these questions, the first affirmatively and the second negatively. We also give an example where one has $\mathcal{E}_{k}(T)^{\perp} \neq \mathcal{E}_{k}\left(T^{2}\right)^{\perp}$, for $k \geq 2$.

The following is our main result and gives a positive answer to the first question of Lesigne:

Theorem 1.3. Let $(X, \mathcal{X}, \mu, T)$ be a totally ergodic system, $f \in L^{1}(\mu)$, and $k \in \mathbb{N}$. Then the following three conditions are equivalent:

(i) $f \in \mathcal{E}_{k}(T)^{\perp}$.

\footnotetext{
${ }^{1}$ Abramov studied in 1 totally ergodic systems for which the subspace spanned by elements of $\bigcup_{k \in \mathbb{N}} \mathcal{E}_{k}(T)$ is dense in $L^{2}(\mu)$. He showed that any such system is isomorphic to a unipotent affine transformation on a compact Abelian group.
} 
(ii) For a.e. $x \in X$, we have for every continuous $\phi: \mathbb{T} \rightarrow \mathbb{C}$, and $P \in \mathbb{R}_{k}[t]$ that

$$
\lim _{N \rightarrow \infty} \frac{1}{N} \sum_{n=1}^{N} \phi(P(n)) \cdot f\left(T^{n} x\right)=0 .
$$

(iii) For a.e. $x \in X$, we have for every continuous $\phi: \mathbb{T} \rightarrow \mathbb{C}$ that

$$
\lim _{N \rightarrow \infty} \sup _{P \in \mathbb{R}_{k}[t]}\left|\frac{1}{N} \sum_{n=1}^{N} \phi(P(n)) \cdot f\left(T^{n} x\right)\right|=0 .
$$

We stress that in $(i i)$ the set of full measure does not depend on the choice of the function $\phi$ or the polynomial $P \in \mathbb{R}_{k}[t]$, and in (iii) on the choice of the function $\phi$.

The following result gives a negative answer to the second question of Lesigne:

Theorem 1.4. (i) For $k \geq 2$, there exists an ergodic system $(X, \mathcal{B}, \mu, T)$ such that $\mathcal{E}_{k}(T)^{\perp} \neq \mathcal{E}_{k}\left(T^{2}\right)^{\perp}$.

(ii) For $k \geq 2$, Theorem 1.3 and part (ii) of Theorem 1.2 fail if we replace the total ergodicity assumption with ergodicity.

In Section 2 we prove Theorem 1.3 by combining the method used in [4] to prove the nonuniform result, with a new elementary idea that enables us to get uniformity (this is Proposition 2.3 but the main idea is contained in Lemma 2.2). Finally, in Section 3 we prove Theorem 1.4. We construct the system explicitly as a skew product extension of a group rotation.

Acknowledgments: I would like to thank J. Campbell for bringing to my attention the questions studied in this article. I would also like to thank B. Kra and E. Lesigne for helpful comments.

\section{Proof of the uniform Polynomial Wiener-Wintner}

We will prove Theorem 1.3. We start with a reduction:

Proposition 2.1. It suffices to check the implication $(i) \Rightarrow$ (iii) in Theorem 1.3 when $\phi(t)=e(t)$ and $f \in L^{\infty}(\mu)$.

Proof. Since every continuous function on $\mathbb{T}$ can be uniformly approximated by trigonometric polynomials we see that it suffices to verify (5) for $\phi(t)=e(m t), m \in \mathbb{Z}$. Furthermore, since for every $m \in \mathbb{Z}$ we have $m P \in \mathbb{R}_{k}[t]$ whenever $P \in \mathbb{R}_{k}[t]$, we can restrict ourselves to the case where $m=1$.

Suppose that for every $f \in L^{\infty}(\mu)$ the implication $(i) \Rightarrow($ iii $)$ in Theorem 1.3 holds, we claim that it also holds for every $f \in L^{1}(\mu)$. Let $k \in \mathbb{N}$ and $f \in L^{1}(\mu)$ be such that $f \in \mathcal{E}_{k}(T)^{\perp}$. We first notice that there exists a sequence of functions $\left\{f_{l}\right\}_{l \in \mathbb{N}}$ such that $f_{l} \in L^{\infty}(\mu), f_{l} \in \mathcal{E}_{k}(T)^{\perp}$, and $\left\|f-f_{l}\right\|_{L^{1}(\mu)} \leq 1 / l$. To see this, we choose $g_{l} \in L^{\infty}(\mu)$ such that $\left\|f-g_{l}\right\|_{L^{1}(\mu)} \leq 1 /(2 l)$. Let $\mathcal{D}$ be the $\sigma$-algebra generated by all $h$-measurable sets where $h \in \mathcal{E}_{k}(T)$. Since $\mathbb{E}(f \mid \mathcal{D})=0$ and $\left\|f-g_{l}\right\|_{L^{1}(\mu)} \leq 1 /(2 l)$, we 
have $\left\|\mathbb{E}\left(g_{l} \mid \mathcal{D}\right)\right\|_{L^{1}(\mu)} \leq 1 /(2 l)$. It is now easy to check that the sequence $\left\{f_{l}\right\}_{l \in \mathbb{N}}$, where $f_{l}=g_{l}-\mathbb{E}\left(g_{l} \mid \mathcal{D}\right)$, satisfies the advertised conditions.

Let $X_{0}$ be the set of $x \in X$ for which the ergodic theorem holds for functions of the form $f_{l}-f$, for all $l \in \mathbb{N}$. Obviously $m\left(X_{0}\right)=1$. For every $x \in X$ we have

$$
\lim _{N \rightarrow \infty} \sup _{P \in \mathbb{R}_{k}[t]}\left|\frac{1}{N} \sum_{n=1}^{N} e(P(n)) \cdot f\left(T^{n} x\right)\right| \leq A_{l}+B_{l},
$$

where

$$
A_{l}=\lim _{N \rightarrow \infty} \sup _{P \in \mathbb{R}_{k}[t]}\left|\frac{1}{N} \sum_{n=1}^{N} e(P(n)) \cdot\left(f\left(T^{n} x\right)-f_{l}\left(T^{n} x\right)\right)\right|
$$

and

$$
B_{l}=\lim _{N \rightarrow \infty} \sup _{P \in \mathbb{R}_{k}[t]}\left|\frac{1}{N} \sum_{n=1}^{N} e(P(n)) \cdot f_{l}\left(T^{n} x\right)\right| .
$$

For $x \in X_{0}$ we have

$$
A_{l} \leq \lim _{N \rightarrow \infty} \frac{1}{N} \sum_{n=1}^{N}\left|f\left(T^{n} x\right)-f_{l}\left(T^{n} x\right)\right|=\int\left|f(x)-f_{l}(x)\right| d \mu \leq 1 / l .
$$

We also know that there exists a set of full measure $X_{1}$ such that $B_{l}=0$ for $x \in X_{1}$. Set $X_{2}=X_{0} \cap X_{1}$. Letting $l \rightarrow \infty$ in (6) gives that for $x \in X_{2}$ we have

$$
\lim _{N \rightarrow \infty} \sup _{P \in \mathbb{R}_{k}[t]}\left|\frac{1}{N} \sum_{n=1}^{N} e(P(n)) \cdot f\left(T^{n} x\right)\right|=0
$$

Since $X_{2}$ has full measure the claim is proved.

The following "uniformization" trick contains the main idea needed for Proposition 2.3, which in turn is one of the key ingredients needed for the proof of Theorem 1.3. We prove it in order to make the argument of Proposition 2.3 more transparent.

Lemma 2.2. Let $b(n)$ be a bounded sequence of complex numbers, such that for every $\alpha \in[0,1]$ we have

$$
\lim _{M \rightarrow \infty} \limsup _{N \rightarrow \infty} \frac{1}{N} \sum_{n=1}^{N}\left|\frac{1}{M} \sum_{m=1}^{M} e(m \alpha) \cdot b(M n+m)\right|=0 .
$$

Then

$$
\lim _{N \rightarrow \infty} \sup _{\alpha \in[0,1]}\left|\frac{1}{N} \sum_{n=1}^{N} e(n \alpha) \cdot b(n)\right|=0 .
$$


Proof. Let $\varepsilon>0$. We can assume that $b(n)$ is bounded by 1 . Then for every $M, N \in \mathbb{N}$ and $\alpha, \beta \in[0,1]$, we have

$$
\begin{aligned}
\left|\frac{1}{N} \sum_{n=1}^{N} e(n \beta) \cdot b(n)\right| & \leq \frac{1}{[N / M]} \sum_{n=1}^{[N / M]}\left|\frac{1}{M} \sum_{m=1}^{M} e(m \beta) \cdot b(M n+m)\right|+2 M / N \\
& \leq A_{M, N}+B_{M, N}
\end{aligned}
$$

where

$$
A_{M, N}=\frac{1}{[N / M]} \sum_{n=1}^{[N / M]} \frac{1}{M} \sum_{m=1}^{M}|e(m \beta)-e(m \alpha)|
$$

and

$$
B_{M, N}=\frac{1}{[N / M]} \sum_{n=1}^{[N / M]}\left|\frac{1}{M} \sum_{m=1}^{M} e(m \alpha) \cdot b(M n+m)\right|+2 M / N .
$$

It follows from (7), that for fixed $\alpha \in[0,1]$, we can choose $M_{\alpha}$ and $N_{\alpha}$ such that for all $N>N_{\alpha}$ we have $B_{M_{\alpha}, N} \leq \varepsilon / 2$. We can choose a neighborhood $V_{\alpha}$ of $\alpha$ such that $\sup _{\beta \in V_{\alpha}, 1 \leq m \leq M_{\alpha}}|e(m \beta)-e(m \alpha)| \leq \varepsilon / 2$. Then for every $N \in \mathbb{N}$ and $\beta \in V_{\alpha}$ we have $A_{M_{a}, N} \leq \varepsilon / 2$. Putting these two estimates together we get that if $N>N_{\alpha}$ then

$$
\sup _{\beta \in V_{\alpha}}\left|\frac{1}{N} \sum_{n=1}^{N} e(n \beta) \cdot b(n)\right| \leq \varepsilon
$$

Doing this for every $\alpha \in[0,1]$, we produce an open cover $\left\{V_{\alpha}\right\}_{\alpha \in[0,1]}$ of $[0,1]$ and positive integers $\left\{N_{\alpha}\right\}_{\alpha \in[0,1]}$, such that (9) holds for $N>N_{\alpha}$. By compactness, there exists a finite subcover, say $V_{\alpha_{1}}, \ldots, V_{\alpha_{l}}$. Then for $N>\max \left\{N_{\alpha_{1}}, \ldots, N_{\alpha_{l}}\right\}$ we have

$$
\sup _{\alpha \in[0,1]}\left|\frac{1}{N} \sum_{n=1}^{N} e(n \alpha) \cdot b(n)\right| \leq \varepsilon .
$$

This proves (8) .

Proposition 2.3. Let $(X, \mathcal{X}, \mu, T)$ be a totally ergodic system, and $f \in L^{\infty}(\mu)$ be such that for every $\alpha \in \mathbb{R}$ we have that for a.e. $x \in X$ (the set of full measure may depend on the choice of $\alpha$ )

$$
\lim _{N \rightarrow \infty} \sup _{P \in \mathbb{R}_{k-1}[t]}\left|\frac{1}{N} \sum_{n=1}^{N} e\left(n^{k} \alpha+P(n)\right) \cdot f\left(T^{n} x\right)\right|=0 .
$$

Then for a.e. $x \in X$ we have

$$
\lim _{N \rightarrow \infty} \sup _{P \in \mathbb{R}_{k}[t]}\left|\frac{1}{N} \sum_{n=1}^{N} e(P(n)) \cdot f\left(T^{n} x\right)\right|=0
$$


Proof. Let $f \in L^{\infty}(\mu)$. Without loss of generality we can assume that $|f(x)| \leq 1$ for every $x \in X$.

First step. Motivated by Lemma 2.2 we first show that there exists a set of full measure $X_{0}$, such that for $x \in X_{0}$, and for every $\alpha \in \mathbb{R}$ we have

$$
\lim _{M \rightarrow \infty} \lim _{N \rightarrow \infty} \frac{1}{N} \sum_{n=1}^{N} \sup _{P \in \mathbb{R}_{k-1}[t]}\left|\frac{1}{M} \sum_{m=1}^{M} e\left(m^{k} \alpha+P(m)\right) \cdot f\left(T^{M n+m} x\right)\right|=0 .
$$

To see this, for $M \in \mathbb{N}$ and $\alpha \in \mathbb{Q}$, we apply the ergodic theorem for the transformation $T^{M}$ (we use total ergodicity here) and the function

$$
g_{M, \alpha}(x)=\sup _{P \in \mathbb{R}_{k-1}[t]}\left|\frac{1}{M} \sum_{m=1}^{M} e\left(m^{k} \alpha+P(m)\right) \cdot f\left(T^{m} x\right)\right| .
$$

Since we are interested in only countably many functions and transformations, we get a set $X_{0}$ of full measure, such that for every $x \in X_{0}, M \in \mathbb{N}$, and $\alpha \in \mathbb{Q}$ we have

$$
\begin{gathered}
\lim _{N \rightarrow \infty} \frac{1}{N} \sum_{n=1}^{N} \sup _{P \in \mathbb{R}_{k-1}[t]}\left|\frac{1}{M} \sum_{m=1}^{M} e\left(m^{k} \alpha+P(m)\right) \cdot f\left(T^{M n+m} x\right)\right|= \\
\int \sup _{P \in \mathbb{R}_{k-1}[t]}\left|\frac{1}{M} \sum_{m=1}^{M} e\left(m^{k} \alpha+P(m)\right) \cdot f\left(T^{m} x\right)\right| d \mu .
\end{gathered}
$$

Since the set of $\alpha \in \mathbb{R}$ for which (13) holds is closed, we get that it holds for all $\alpha \in \mathbb{R}$, $x \in X_{0}$, and $M \in \mathbb{N}$. The claim now follows by letting $M \rightarrow \infty$ in (13), using our assumption (10), and the bounded convergence theorem.

Second step. Fix $x \in X_{0}$ and $\varepsilon>0$. We claim that for every $\alpha \in \mathbb{R}$ there exists $N_{\alpha} \in \mathbb{N}$ and open neighborhood $V_{\alpha}$ of $\alpha\left(N_{\alpha}\right.$ and $V_{\alpha}$ also depend on $x$ and $\left.\varepsilon\right)$, such that for every $N>N_{\alpha}$ we have

$$
\sup _{P \in \mathbb{R}_{k-1}[t], \beta \in V_{\alpha}}\left|\frac{1}{N} \sum_{n=1}^{N} e\left(n^{k} \beta+P(n)\right) \cdot f\left(T^{n} x\right)\right| \leq \varepsilon .
$$

So let $\alpha \in \mathbb{R}$. If $P \in \mathbb{R}_{k-1}[t]$, introducing an error term $C_{M, N}$ that satisfies $\left|C_{M, N}\right| \leq$ $2 M / N$ we have

$$
\begin{gathered}
\frac{1}{N} \sum_{n=1}^{N} e\left(n^{k} \beta+P(n)\right) \cdot f\left(T^{n} x\right)= \\
\frac{1}{[N / M]} \sum_{n=1}^{[N / M]} \frac{1}{M} \sum_{m=1}^{M} e\left((M n+m)^{k} \beta+P(M n+m)\right) \cdot f\left(T^{M n+m} x\right)+C_{M, N}= \\
A_{M, N}+B_{M, N}+C_{M, N},
\end{gathered}
$$


where

$$
A_{M, N}=\frac{1}{[N / M]} \sum_{n=1}^{[N / M]} \frac{1}{M} \sum_{m=1}^{M}\left(e\left(m^{k} \beta+P_{M, n, \beta}(m)\right)-e\left(m^{k} \alpha+P_{M, n, \beta}(m)\right)\right) \cdot f\left(T^{M n+m} x\right)
$$

for some $P_{M, n, \beta} \in \mathbb{R}_{k-1}[t]$, and

$$
B_{M, N}=\frac{1}{[N / M]} \sum_{n=1}^{[N / M]} \frac{1}{M} \sum_{m=1}^{M} e\left(m^{k} \alpha+P_{M, n, \beta}(m)\right) \cdot f\left(T^{M n+m} x\right) .
$$

By (12), there exists $M_{\alpha} \in \mathbb{N}$ such that if $N$ is large enough we have

$$
\left|B_{M_{\alpha}, N}\right| \leq \frac{1}{\left[N / M_{\alpha}\right]} \sum_{n=1}^{\left[N / M_{\alpha}\right]} \sup _{P \in \mathbb{R}_{k-1}[t]}\left|\frac{1}{M_{\alpha}} \sum_{m=1}^{M_{\alpha}} e\left(m^{k} \alpha+P(m)\right) \cdot f\left(T^{M_{\alpha} n+m} x\right)\right| \leq \varepsilon / 3 .
$$

Choose a neighborhood $V_{\alpha}$ of $\alpha$ such that

$$
\sup _{\beta \in V_{\alpha}, 1 \leq m \leq M_{\alpha}}\left|e\left(m^{k} \alpha\right)-e\left(m^{k} \beta\right)\right| \leq \varepsilon / 3 .
$$

Then for $\beta \in V_{\alpha}$ we have

$$
\left|A_{M_{\alpha}, N}\right| \leq \frac{1}{\left[N / M_{\alpha}\right]} \sum_{n=1}^{\left[N / M_{\alpha}\right]} \frac{1}{M_{\alpha}} \sum_{m=1}^{M_{\alpha}}\left|e\left(m^{k} \beta\right)-e\left(m^{k} \alpha\right)\right| \leq \varepsilon / 3 .
$$

Putting all these estimates together we see that if $N$ is large enough, for all $P \in \mathbb{R}_{k-1}[t]$ and $\beta \in V_{\alpha}$ the average in (15) is bounded in absolute value by $2 \varepsilon / 3+M_{\alpha} / N \leq \varepsilon$, proving our claim.

Third step. We finish the proof. Notice first that since the function $e(t)$ is 1-periodic it suffices to verify (11) when the sup is taken over all polynomials with leading term belonging to the interval $[0,1]$. Let $\varepsilon>0$ and $x \in X_{0}$. Let $V_{\alpha}$ be the open neighborhood of $\alpha \in[0,1]$ and $N_{a}$ be the positive integer for which (14) holds. Since $\left\{V_{\alpha}\right\}_{\alpha \in[0,1]}$ is an open cover of the compact set $[0,1]$ there exists a finite subcover, say $\left\{V_{\alpha_{i}}\right\}_{i=1, \ldots l}$. Then for every $N>N_{0}=\max \left\{N_{a_{1}}, \ldots, N_{a_{l}}\right\}$ we have

$$
\sup _{P \in \mathbb{R}_{k-1}[t], \alpha \in[0,1]}\left|\frac{1}{N} \sum_{n=1}^{N} e\left(n^{k} \alpha+P(n)\right) \cdot f\left(T^{n} x\right)\right| \leq \varepsilon .
$$

So (11) holds for every $x \in X_{0}$, completing the proof of the lemma.

Notice that for totally ergodic systems, the case $k=1$ of Proposition 2.3 gives yet another proof of the uniform Wiener-Wintner theorem (see (ii) in page 1).

We now combine the argument in [4] with the previous Proposition to prove Theorem 1.3 . 
Proof of Theorem 1.3. The implication $($ iii $) \Rightarrow(i i)$ is obvious. The implication $(i i) \Rightarrow(i)$ is easy and was proved in [4], but we reprove it for completeness. Let $g \in \mathcal{E}_{k}(T)$, and $f \in L^{1}(\mu)$ that satisfies condition $(i i)$ of Theorem 1.3. It is easy to check that

$$
g\left(T^{n} x\right)=e\left(P_{x}(n)\right)
$$

for some $P_{x} \in \mathbb{R}_{k}[t]$, and by assumption for a.e. $x \in X$ we have for every $P \in \mathbb{R}_{k}[t]$ that

$$
\lim _{N \rightarrow \infty} \frac{1}{N} \sum_{n=1}^{N} e(P(n)) \cdot f\left(T^{n} x\right)=0 .
$$

By the ergodic theorem and (16) we have for a.e. $x \in X$ that

$$
\int g f d \mu=\lim _{N \rightarrow \infty} \frac{1}{N} \sum_{n=1}^{N} g\left(T^{n} x\right) \cdot f\left(T^{n} x\right)=\lim _{N \rightarrow \infty} \frac{1}{N} \sum_{n=1}^{N} e\left(P_{x}(n)\right) \cdot f\left(T^{n} x\right),
$$

and this is 0 by (17). Hence, $f$ is orthogonal to $g$, showing that $f \in \mathcal{E}(T)^{\perp}$.

So it remains to show the implication $(i) \Rightarrow($ iii $)$. By Proposition 2.1 we can restrict ourselves to the case where $f \in L^{\infty}(\mu)$ and $\phi(t)=e(t)$. We proceed in two steps:

First Step. Suppose that $\alpha \in E_{0}^{\prime}$, where $E_{0}^{\prime}$ is the set of $\alpha \in \mathbb{R}$ such that $e(m \alpha) \notin$ $\mathcal{E}_{0}(T)$ for every nonzero integer $m$. We claim that for every $k \in \mathbb{N}$ and $f \in L^{\infty}(\mu)$, we have for a.e. $x \in X$ (the set of full measure may depend on $\alpha$ ) that

$$
\lim _{N \rightarrow \infty} \sup _{P \in \mathbb{R}_{k-1}[t]}\left|\frac{1}{N} \sum_{n=1}^{N} e\left(n^{k} \alpha+P(n)\right) \cdot f\left(T^{n} x\right)\right|=0 .
$$

We will use induction on $k$. For $k=1$ the statement follows by applying the ergodic theorem for the system $\left(X \times \mathbb{T}, \mathcal{X} \times \mathcal{B}, \mu \times m, T \times R_{\alpha}\right)$, and the function $g(x, t)=f(x) \cdot e(t)$, where $\mathcal{B}$ is the Borel $\sigma$-algebra, $m$ is the Haar measure on $\mathbb{T}$, and $R_{\alpha}(t)=t+\alpha$ (the system is ergodic because $\alpha \in E_{0}^{\prime}$ ). Suppose that the statement holds for $k-1$, we will show that it holds for $k$. Using van der Corput's Lemma ${ }^{2}$ (see 2]) for the sequence $a_{x}(n)=e\left(n^{k} \alpha+P(n)\right) \cdot f\left(T^{n} x\right)$, we find that for every $x \in X$ and integers $1 \leq H \leq N$ we have

$$
\sup _{P \in \mathbb{R}_{k-1}[t]}\left|\frac{1}{N} \sum_{n=1}^{N} e\left(n^{k} \alpha+P(n)\right) \cdot f\left(T^{n} x\right)\right|^{2} \leq \frac{N+H}{N(H+1)} \cdot \frac{1}{N} \sum_{n=1}^{N}\left|f\left(T^{n} x\right)\right|^{2}+
$$

$2 \frac{N+H}{N(H+1)^{2}} \sum_{h=1}^{H}(H+1-h) \cdot \sup _{Q \in \mathbb{R}_{k-2}[t]}\left|\frac{1}{N} \sum_{n=1}^{N-h} e\left(k h n^{k-1} \alpha+Q(n)\right) \cdot f\left(T^{n+h} x\right) \cdot \overline{f\left(T^{n} x\right)}\right|$.

\footnotetext{
${ }^{2}$ This says that if $a(n)$ is a sequence of complex numbers then for all integers $1 \leq H \leq N$ we have $\left|\frac{1}{N} \sum_{n=1}^{N} a(n)\right|^{2} \leq \frac{N+H}{N(H+1)} \cdot \frac{1}{N} \sum_{n=1}^{N}|a(n)|^{2}+2 \frac{N+H}{N(H+1)^{2}} \sum_{h=1}^{H}(H+1-h) \cdot \operatorname{Re}\left(\frac{1}{N} \sum_{n=1}^{N-h} a(n+h) \cdot \overline{a(n)}\right)$.
} 
We let $N \rightarrow \infty$ in (19). Using the ergodic theorem for the function $|f|^{2}$, and the induction hypothesis for the values $k h \alpha \in E_{0}^{\prime}$ and the functions $T^{h} f \cdot f$, for $h \in \mathbb{N}$, we find that for every $H \in \mathbb{N}$ we have for a.e. $x \in X$ that

$$
\lim _{N \rightarrow \infty} \sup _{P \in \mathbb{R}_{k-1}[t]}\left|\frac{1}{N} \sum_{n=1}^{N} e\left(n^{k} \alpha+P(n)\right) \cdot f\left(T^{n} x\right)\right|^{2} \leq \frac{1}{H+1} \int|f(x)|^{2} d \mu .
$$

Letting $H \rightarrow \infty$ we get (18), proving the claim.

Second Step. We now prove (5). We will use induction on $k$. For $k=1$ the statement is known to be true for all ergodic systems (see $(i)$ and (ii) in page 1). Suppose that the statement holds for $k-1 \geq 1$, we will show that it holds for $k \geq 2$. By Proposition 2.3 it suffices to show that for every $\alpha \in \mathbb{R}$, we have for a.e. $x \in X$ that

$$
\lim _{N \rightarrow \infty} \sup _{P \in \mathbb{R}_{k-1}[t]}\left|\frac{1}{N} \sum_{n=1}^{N} e\left(n^{k} \alpha+P(n)\right) \cdot f\left(T^{n} x\right)\right|=0 .
$$

We consider two cases:

If $\alpha \in E_{0}^{\prime}$ then we are covered by the first step.

If $\alpha \notin E_{0}^{\prime}$ then $e(m \alpha) \in \mathcal{E}_{0}(T)$ for some nonzero integer $m$. Let $e(\gamma(x))$ be an $e(m \alpha)$ eigenfunction. Consider the system $\left(X \times \mathbb{T}^{k}, \mathcal{X} \times \mathcal{B}_{k}, \mu \times m_{k}, T_{k}\right)$, where $\mathcal{B}_{k}$ is the Borel $\sigma$-algebra, $m_{k}$ is the Haar measure on $\mathbb{T}^{k}$, and

$$
T_{k}\left(x, t_{1}, t_{2}, \ldots, t_{k}\right)=\left(T x, t_{1}+\gamma(x)+b, t_{2}+t_{1}, \ldots, t_{k-1}+t_{k-2}\right) .
$$

As was shown in [4, it is possible to choose $b \in \mathbb{T}$ such that the resulting system is totally ergodic. It was also shown there that for $j \in \mathbb{N}$ every function in $\mathcal{E}_{j}\left(T_{k}\right)$ is a product of a function in $\mathcal{E}_{j+1}(T)$ and a character of $\mathbb{T}^{k}$. It follows that if $g: X \times \mathbb{T}^{k} \rightarrow \mathbb{C}$ is defined by

$$
g\left(x, t_{1}, \ldots, t_{k}\right)=f(x) \cdot e\left(k ! t_{k}\right),
$$

then $g \in \mathcal{E}_{k-1}\left(T_{k}\right)^{\perp}$. So we can apply the inductive hypothesis for the system $(X \times$ $\left.\mathbb{T}^{k}, \mathcal{X} \times \mathcal{B}_{k}, \mu \times m_{k}, T_{k}\right)$ and the function $g$. We get that for a.e. $\left(x, t_{1}, \ldots, t_{k}\right) \in X \times \mathbb{T}^{k}$ we have

$$
\lim _{N \rightarrow \infty} \sup _{P \in \mathbb{R}_{k-1}[t]}\left|\frac{1}{N} \sum_{n=1}^{N} e(P(n)) \cdot g\left(T_{k}^{n}\left(x, t_{1}, \ldots, t_{k}\right)\right)\right|=0 .
$$

If $C_{n}^{i}=\left(\begin{array}{c}n \\ i\end{array}\right)$ for $i=1, \ldots, k$, notice that

$$
\begin{aligned}
g\left(T_{k}^{n}\left(x, t_{1}, \ldots, t_{k}\right)\right) & =e\left(k !\left(t_{k}+C_{n}^{1} t_{k-1}+\ldots+C_{n}^{k-2} t_{1}+C_{n}^{k-1}(\gamma(x)+b)+C_{n}^{k} m \alpha\right)\right) \cdot f\left(T^{n} x\right), \\
& =e\left(n^{k} m \alpha+Q_{t_{1}, \ldots, t_{k}, x, b}(n)\right) \cdot f\left(T^{n} x\right),
\end{aligned}
$$

where $Q_{t_{1}, \ldots, t_{k}, x, b} \in \mathbb{R}_{k-1}[t]$. Hence, (21) gives that for a.e. $x \in X$ we have

$$
\lim _{N \rightarrow \infty} \sup _{P \in \mathbb{R}_{k-1}[t]}\left|\frac{1}{N} \sum_{n=1}^{N} e\left(n^{k} m \alpha+P(n)\right) \cdot f\left(T^{n} x\right)\right|=0 .
$$


So in order to get (20), it remains to replace $m \alpha$ by $\alpha$ in (22). We do this as follows: Since $e(m \alpha) \in \mathcal{E}_{0}(T)$ we have $e\left(m^{2} \alpha\right) \in \mathcal{E}_{0}\left(T^{m}\right)$, and since $k \geq 2$ this implies that $e\left(m^{k} \alpha\right) \in \mathcal{E}_{0}\left(T^{m}\right)$. An easy inductive argument (see [4, page 784) shows that for totally ergodic systems $\mathcal{E}_{k}\left(T^{j}\right)=\mathcal{E}_{k}(T)$ for $j \in \mathbb{N}$, so $T^{r} f \in \mathcal{E}_{k}\left(T^{m}\right)^{\perp}$ for every $r \in \mathbb{N}$. Hence, we can repeat the previous argument for the ergodic system $\left(X, \mathcal{B}, \mu, T^{m}\right)$, the eigenvalue $e\left(m^{k} \alpha\right)$ of $T^{m}$, and the functions $T^{r} f, r=0, \ldots, m-1$. We get that for a.e. $x \in X$

$$
\lim _{N \rightarrow \infty} \sup _{P \in \mathbb{R}_{k-1}[t]}\left|\frac{1}{N} \sum_{n=1}^{N} e\left(n^{k} m^{k} \alpha+P(n)\right) \cdot f\left(T^{n m+r} x\right)\right|=0,
$$

for $r=0,1, \ldots, m-1$. This implies that

$$
\lim _{N \rightarrow \infty} \sup _{P \in \mathbb{R}_{k-1}[t]}\left|\frac{1}{N} \sum_{n=1}^{N} e\left((n m+r)^{k} \alpha+P(n m+r)\right) \cdot f\left(T^{n m+r} x\right)\right|=0,
$$

for $r=0,1, \ldots, m-1$, which in turn implies that for a.e. $x \in X$ we have

$$
\lim _{N \rightarrow \infty} \sup _{P \in \mathbb{R}_{k-1}[t]}\left|\frac{1}{N} \sum_{n=1}^{N} e\left(n^{k} \alpha+P(n)\right) \cdot f\left(T^{n} x\right)\right|=0 .
$$

This proves (201) and completes the proof of the induction step.

\section{Two Counterexamples.}

We construct an ergodic system $(X, \mathcal{B}, \mu, T)$ for which $\mathcal{E}_{k}(T)^{\perp} \neq \mathcal{E}_{k}\left(T^{2}\right)^{\perp}$ for $k \geq 2$, and for which part (ii) of Theorem 1.2 fails.

Proof of Theorem 1.4. (i) It will be clear that for the system we will construct we have $\mathcal{E}_{k}(T)=\mathcal{E}_{2}(T)$ and $\mathcal{E}_{k}\left(T^{2}\right)=\mathcal{E}_{2}\left(T^{2}\right)$ for $k \geq 2$, so we can assume that $k=2$. On $X=\mathbb{Z}_{2} \times \mathbb{T}^{2}$ with the Haar measure $\mu$, consider the measure preserving transformation $T: X \rightarrow X$ defined by

$$
T\left(0, t_{1}, t_{2}\right)=\left(1, t_{1}, t_{2}\right), \quad T\left(1, t_{1}, t_{2}\right)=\left(0, t_{1}+\alpha, t_{2}+t_{1}\right)
$$

for some $\alpha \in \mathbb{R}$ irrational. We can see that $T$ is ergodic as follows: We have

$$
T^{2}\left(i, t_{1}, t_{2}\right)=\left(i, t_{1}+\alpha, t_{2}+t_{1}\right)
$$

and since the skew product transformation $S\left(t_{1}, t_{2}\right)=\left(t_{1}+\alpha, t_{2}+t_{1}\right)$ defined on $\mathbb{T}^{2}$ with the Haar measure is known to be ergodic for $\alpha$ irrational, we have that $T^{2}$ has two ergodic components, the sets $X_{i}=\{i\} \times \mathbb{T}^{2}, i=1$, . Since neither of these sets is $T$-invariant, the transformation $T$ is ergodic.

It is clear that $e\left(t_{2}\right) \in \mathcal{E}_{2}\left(T^{2}\right)$. We will show that $e\left(t_{2}\right) \in \mathcal{E}_{2}(T)^{\perp}$. First we compute $\mathcal{E}_{1}(T)$. Let $h \in \mathcal{E}_{1}(T)$, then

$$
h\left(T\left(i, t_{1}, t_{2}\right)\right)=c h\left(i, t_{1}, t_{2}\right)
$$


for some nonzero $c \in \mathbb{C}$. A standard Fourier series argument shows that $h$ does not depend on $t_{2}$, so $h\left(i, t_{1}, t_{2}\right)=h_{1}\left(i, t_{1}\right)$. Then (23) takes the form

$$
h_{1}\left(1, t_{1}\right)=c h_{1}\left(0, t_{1}\right), \quad h_{1}\left(0, t_{1}+\alpha\right)=c h_{1}\left(1, t_{1}\right) .
$$

Substituting the first equation into the second gives

$$
h_{1}\left(0, t_{1}+\alpha\right)=c^{2} h_{1}\left(0, t_{1}\right) .
$$

This easily implies that for some $m \in \mathbb{Z}$ and nonzero $c_{1} \in \mathbb{C}$ we have

$$
h\left(0, t_{1}, t_{2}\right)=c_{1} e\left(m t_{1}\right), \quad h\left(1, t_{1}, t_{2}\right)=c_{2} e\left(m t_{1}\right),
$$

where $c_{2}= \pm c_{1} e(m \alpha / 2)$.

Next we show that $e\left(t_{2}\right) \in \mathcal{E}_{2}(T)^{\perp}$. Let $f \in \mathcal{E}_{2}(T)$, then there exists $h \in \mathcal{E}_{1}(T)$ such that

$$
f\left(T\left(i, t_{1}, t_{2}\right)\right)=h\left(i, t_{1}, t_{2}\right) \cdot f\left(i, t_{1}, t_{2}\right) .
$$

A standard Fourier series argument gives that

$$
f\left(i, t_{1}, t_{2}\right)=e\left(l t_{2}\right) \cdot g\left(i, t_{1}\right)
$$

for some $l \in \mathbb{Z}$. Substituting this into (25) and using (24) gives

$$
\begin{gathered}
g\left(1, t_{1}\right)=c_{1} e\left(m t_{1}\right) \cdot g\left(0, t_{1}\right), \\
g\left(0, t_{1}+\alpha\right) \cdot e\left(l t_{2}\right)=c_{2} t e\left(m t_{1}\right) \cdot g\left(1, t_{1}\right),
\end{gathered}
$$

for some $m \in \mathbb{Z}$ and nonzero $c_{1}, c_{2} \in \mathbb{C}$. Substituting the first equation into the second gives

$$
g\left(0, t_{1}+\alpha\right)=c_{3} e\left((2 m-l) t_{1}\right) \cdot g\left(0, t_{1}\right)
$$

where $c_{3}=c_{1} c_{2} \neq 0$. The last equation has a solution only if $l=2 m$. Combining this with (26) gives that every $f \in \mathcal{E}_{2}(T)$ has the form

$$
f\left(i, t_{1}, t_{2}\right)=e\left(2 m t_{1}\right) \cdot g\left(i, t_{1}\right)
$$

for some $m \in \mathbb{Z}$. This shows that $e\left(t_{2}\right) \in \mathcal{E}_{2}(T)^{\perp}$ and completes the proof of the first part of Theorem 1.4

(ii) It suffices to construct an ergodic system $(X, \mathcal{B}, \mu, T)$, a function $f \in L^{\infty}(\mu)$ with $f \in \mathcal{E}_{2}(T)^{\perp}$, and a set $X_{1} \subset X$ with $\mu\left(X_{1}\right)>0$, and such that for every $x \in X_{1}$ there exists $P_{x} \in \mathbb{R}_{2}[t]$ satisfying

$$
\lim _{N \rightarrow \infty} \frac{1}{N} \sum_{n=1}^{N} e\left(P_{x}(n)\right) \cdot f\left(T^{n} x\right) \neq 0 .
$$


We use the measure preserving system constructed in (i). We showed before that $f\left(i, t_{1}, t_{2}\right)=e\left(t_{2}\right) \in \mathcal{E}_{2}(T)^{\perp}$. Notice that for $n \in \mathbb{N}$ we have

$$
\begin{gathered}
T^{2 n}\left(1, t_{1}, t_{2}\right)=\left(1, t_{1}+n \alpha, t_{2}+n t_{1}+C_{n}^{2} \alpha\right), \\
T^{2 n+1}\left(1, t_{1}, t_{2}\right)=\left(0, t_{1}+(n+1) \alpha, t_{2}+(n+1) t_{1}+C_{n+1}^{2} \alpha\right),
\end{gathered}
$$

where $C_{n}^{2}=\left(\begin{array}{l}n \\ 2\end{array}\right)$. Let $X_{1}=\{1\} \times \mathbb{T}^{2}$, then $\mu\left(X_{1}\right)=1 / 2$. If $x=\left(1, t_{1}, t_{2}\right) \in X_{1}$ and $P_{x} \in \mathbb{R}_{2}[t]$ is such that

then for every $n \in \mathbb{N}$

$$
P_{x}(2 t)=e\left(-t_{2}-t t_{1}-\frac{t(t-1)}{2} \alpha\right)
$$

$$
e\left(P_{x}(2 n)\right) \cdot f\left(T^{2 n}\left(1, t_{1}, t_{2}\right)\right)=1, \quad e\left(P_{x}(2 n+1)\right) \cdot f\left(T^{2 n+1}\left(1, t_{1}, t_{2}\right)\right)=c_{t_{1}} e(n \alpha / 2)
$$

where $c_{t_{1}}=e\left(t_{1} / 2+\alpha / 8\right)$. It follows that (27) holds for $x \in X_{1}$, completing the proof of the second part of Theorem 1.4 .

\section{REFERENCES}

[1] L. M. Abramov. Metric automorphisms with quasi-discrete spectrum. Izv. Akad. Nauk SSSR Ser. Mat., 26, (1962), 513-530.

[2] L. Kuipers and H. Niederreitter. Uniform distribution of sequences. Wiley, New York, (1974).

[3] E. Lesigne. Un théorème de disjonction de systèmes dynamiques et une généralisation du théorème ergodique de Wiener-Wintner. Ergod. Th. 83 Dynam. Sys., 10, (1990), 513-521.

[4] E. Lesigne, Spectre quasi-discret et théorème ergodique de Wiener-Wintner pour les polynômes. Ergod. Th. \& Dynam. Sys., 13 (1993), 767-784.

[5] N. Wiener \& A. Wintner. Harmonic analysis and ergodic theory. Amer. J. Math., 63, (1941), $415-426$.

1 Einstein Drive, Institute for Advanced Study, Princeton, NJ 08540 